BULL. AUSTRAL. MATH. SOC.

VOL. $2(1970), 142-143$.

\title{
Smooth semi-inner-product spaces
}

\author{
J. R. Giles
}

With a semi-inner-product on a normed linear space is associated a certain mapping from the space into its dual, called a support mapping. It is shown that properties of the support mapping are closely allied to the Gâteaux differentials of the norm, and that the various differentiability properties for the norm can be characterised by corresponding continuity properties for the support mappings. Continuity properties of the support mappings used in conjunction with the subreflexivity of Banach spaces provide a simple technique in establishing many results which involve differentiability of the norm.

Using the fact that smoothness is characterised by a continuity property of the semi-inner-product, inner product arguments are extended to the study of the geometry of smooth semi-inner-product spaces. Rotundity can be expressed readily in terms of the semi-inner-product. A generalised orthogonality relation is defined in terms of the semi-inner-product and is used to give a representation theorem for continuous linear functionals similar to the Riesz representation theorem for Hilbert space. The semi-inner-product representation of the dual space is studied and questions of the duality of smoothness and rotundity are discussed. The class of uniform semi-inner-product spaces is defined and attention drawn to the duality properties of such spaces.

H. Rund's approach to the differential geometry of Minkowskian space was a motivating influence in the research for the thesis. In establishing the uniform semi-inner-product character of a smooth and rotund finite dimensional normed linear space it is shown that many of Rund's results are independent of the context of classical differential geometry.

An analysis of $L_{p}$-spaces, where $l<p<\infty$, as uniform

Received 17 April 1969. Thesis submitted to the University of Newcastle, December 1968. Degree approved, March 1969. Supervisor: Professor G. Szekeres. 
semi-inner-product spaces illustrates the facility of the theory in handling duality questions.

An application of support mapping properties is given in an examination of part of the theory of the numerical range of an operator on a normed linear space as developed by G. Lumer and F.F. Bonsall.

Several areas for possible further research are outlined.

The following papers have arisen from the research for the thesis:

J.R. Giles, "Classes of semi-inner-product spaces", Trans. Amer. Math. Soc. 129 (1967), 436-446.

J.R. Giles, "On a characterisation of differentiability of the norm of a normed linear space", J. Austral. Math. Soc. (to appear). 